DOI: $10.17516 / 1997-1397-2021-14-2-184-192$

УДК 517.9

\title{
Supercritical Convection of Water in an Elongated Cavity at a Given Vertical Heat Flux
}

\author{
Vadim A. Sharifulin* \\ Perm National Research Polytechnic University \\ Perm, Russian Federation \\ Tatyana P. Lyubimova ${ }^{\dagger}$ \\ Institute of Continuous Media Mechanics Ural Branch of RAS \\ Perm, Russian Federation
}

Received 14.06.2020, received in revised form 11.10.2020, accepted 22.11.2020

\begin{abstract}
The supercritical modes of water convection are investigated at room temperature in an elongated horizontal cavityes, with a width-to-height ratios of $2: 1$ and $3: 1$. The Prandtl number is assumed to be equal to seven. A constant heat flux is set at the upper free and lower solid boundaries, and the lateral solid boundaries are assumed to be thermally insulated. Calculations carried out by the finite-difference method for values of the Rayleigh number exceeding the critical one by up to thirty times have shown that in the indicated interval of Rayleigh numbers in both cavities in the supercritical region, a single-vortex steady state is realized.
\end{abstract}

Keywords: thermal convection, bifurcations, fixed heat flux.

Citation: V.A. Sharifulin, T.P. Lyubimova, Supercritical Convection of Water in an Elongated Cavity at a Given Vertical Heat Flux, J. Sib. Fed. Univ. Math. Phys., 2021, 14(2), 184-192.

DOI: $10.17516 / 1997-1397-2021-14-2-184-192$.

To optimize technological processes with the participation of fluidsystems, it is desirable to develop methods for controlling the structure of the movement of water heated from below, as the most common liquid. A technical reservoir or a natural reservoir can have a free upper boundary, which in a wide range of heating conditions can remain flat and horizontal. With a weak uniform heating from below, when the Rayleigh number $R a$ does not exceed the critical value $R a_{c}$, the liquid can remain at rest. An increase in the intensity of heating $R a>R a_{c}$ leads to Rayleigh-Benard convection, which covers the entire cavity. The form of critical disturbances, the nonlinear development of which leads to a supercritical regime, depends on the conditions of heating (cooling) at the boundaries of the layer. The critical value of the Rayleigh number and the shape of the critical disturbance are determined by the boundary conditions for temperature. So, for isothermal boundaries, the most dangerous disturbance is in the form of cells with a horizontal scale of the order of the layer thickness, and in the case of a given heat flux, longwave disturbances are the most dangerous [1]. The infinity of the layer is a good approximation of an elongated cavity and allows one to determine the threshold for the onset of convection. To study the nonlinear regime arising in the supercritical region, it is necessary to take into account the finiteness of the layer length. Such studies were carried out mainly for the case of isothermal boundaries but, as a rule, in the presence of various complicating factors that

\footnotetext{
*vadsharif@bk.ru https://orcid.org/0000-0001-9932-6622

†lubimova@icmm.ru https://orcid.org/0000-0002-8212-2890

(C) Siberian Federal University. All rights reserved
} 
were important from the point of view of applications. The role of thermal boundary conditions, namely, a given heat flux, remained outside the attention of most researchers. Although with relation to geophysical and technical applications, it is more natural to set not the temperature, but the heat flux at the boundaries [2-4].

In this work, we analyze the influence of the finiteness of the cavity length on the onset of instability and the arising plane supercritical regimes. On the horizontal boundaries (solid bottom and free top), a constant heat flux is set, cavities with length-to-height ratios of $2: 1$ and $3: 1$ are considered. The average temperature in the layer is close to room temperature, the dependence of the density of water on temperature is linear and the Prandtl number $\operatorname{Pr}=7$. Calculations of supercritical flows were carried out in the range of Grashof numbers up to forty supercriticalities $r=R a / R a_{c}$.

\section{Problem formulation}

Consider a horizontally elongated cavity with height $h$ and width $L$, located in an uniform gravity field $g$. The origin of the cartesian coordinate system $(x, z)$ is located at the lower boundary, the $z$ axis is directed vertically upward. The cavity is filled with a viscous incompressible fluid, the lateral and lower boundaries are assumed to be solid, and the upper boundary is free. On both horizontal boundaries $z=0, h$, a fixed vertical heat flux is set $q_{z}=q$, lateral boundaries $x=0, L$ are assumed to be thermally insulated, i.e. the heat flux $q_{x}$ through them is zero.

If the liquid is at rest, then in accordance with the Fourier law, a stationary linear vertical distribution of temperature is established in the cavity, which can be written in the form:

$$
T=T_{0}+A h-A z,
$$

where $A=-q / \kappa$ is the value of a given constant temperature gradient generated by a given heat flux $q, A>0$ corresponds to heating from below, then it will be used as a given external parameter of the problem. We introduce into consideration the temperature $T^{\prime}$ measured from the temperature $T_{0}$, then relation (1) will be rewritten as

$$
T=A h-A z .
$$

Hereinafter, the prime at the temperature is omitted.

The temperature dependence of the density is assumed to be linear, and its changes due to thermal expansion are sufficiently small. It allows one to use the equations of thermal convection in the Boussinesq approximation.

The linear temperature distribution specified in accordance with (2) will change when an instability of the state of mechanical equilibrium arises and the development of a supercritical flow takes place.

The amount of heat entering the layer through the lower boundary per unit time is equal to the heat flux through the upper boundary, as a result of which the average temperature in the layer does not depend on the convection intensity and is determined only by the initial temperature distribution:

$$
T_{a v}=\frac{A h}{2} .
$$

We restrict ourselves to considering plane supercritical motions. Let us introduce the stream function $\psi$ and vorticity $\varphi$ into consideration:

$$
\begin{aligned}
\mathrm{v}_{x}=-\frac{\partial \psi}{\partial z}, \quad \mathrm{v}_{z} & =\frac{\partial \psi}{\partial x}, \quad \varphi=\frac{\partial \mathrm{v}_{x}}{\partial z}-\frac{\partial \mathrm{v}_{z}}{\partial x} . \\
& -185-
\end{aligned}
$$


The equations of free thermal convection of a liquid in the Bussinesq approximation taking into account (4) for variables $(\psi, \varphi, T)$ can be written in dimensionless form [5]:

$$
\begin{gathered}
\frac{\partial \varphi}{\partial t}+\frac{\partial \psi}{\partial x} \frac{\partial \varphi}{\partial z}-\frac{\partial \psi}{\partial z} \frac{\partial \varphi}{\partial x}=\Delta \varphi-\operatorname{Gr} \frac{\partial T}{\partial x} \\
\frac{\partial T}{\partial t}+\frac{\partial \psi}{\partial x} \frac{\partial T}{\partial z}-\frac{\partial \psi}{\partial z} \frac{\partial T}{\partial x}=\frac{1}{\operatorname{Pr}} \Delta T \\
\Delta \psi+\varphi=0
\end{gathered}
$$

The following units of measurement are introduced: time $-h^{2} / \nu$, distance $-h$, temperature $-A h$, velocity $-\nu / h$, stream function $-\nu$ and vorticity $-\nu / h^{2}$. Parameter $\operatorname{Pr}=\nu / \chi$ is the Prandtl number. The dimensionless similarity criterion is an analogue of the Grashof number $G r$, and it is determined through the physical constants of the problem by the relation:

$$
\mathrm{Gr}=\frac{g \beta A h^{4}}{\nu^{2}}
$$

The Rayleigh number is related to $G r$ and $\operatorname{Pr}$ by the ratio:

$$
\mathrm{Ra}=\mathrm{Gr} \cdot \operatorname{Pr}=\frac{g \beta A h^{4}}{\nu \chi}
$$

The coefficients of volumetric expansion $\beta$, kinematic viscosity $\nu$ and thermal diffusivity $\chi$ are assumed to be constant.

The thermocapillary effect, the effects of evaporation and radiation at the upper free boundary are neglected. Then the boundary conditions will be written in the form :

$$
\begin{aligned}
& z=0: \quad \psi=\frac{\partial \psi}{\partial z}=0, \quad \frac{\partial T}{\partial z}=-1, \\
& z=1: \quad \psi=\varphi=0, \quad \frac{\partial T}{\partial z}=-1 .
\end{aligned}
$$

The boundary conditions at solid and thermally insulated side boundaries are written as:

$$
\begin{array}{lll}
x=0: & \psi=\frac{\partial \psi}{\partial x}=0, & \frac{\partial T}{\partial x}=0, \\
x=L: & \psi=\frac{\partial \psi}{\partial x}=0, & \frac{\partial T}{\partial x}=0 .
\end{array}
$$

The state of mechanical equilibrium (2) will be written as:

$$
\psi^{0}=\varphi^{0}=0, \quad T^{0}=1-z .
$$

Thus, the solution to problem (5)-(7), (10)-(11) is determined by the Prandtl number $P r$, Grashof number $G r$ and aspect ratio $L$.

In the dimensionless form condition for the constancy of the average temperature (3) can be written as follows:

$$
T_{a v}=\frac{1}{2} .
$$

Problem (5)-(7), (10)-(11) was solved numerically by the finite-difference method, all spatial derivatives were approximated by central differences on a uniform grid. In most calculations, 
the grid step was assumed to be equal $\Delta=1 / 64$. Cavities with $L=2, L=3$ are considered. An explicit scheme with a constant time step $\Delta^{2} / 10$ was used. The Poisson equation (7) for the stream function was solved on every time step by the succesive over-relaxation method. The vorticity $\varphi$ values on left, right and bottom boundaries were obtained using Thom's formula [6]. The method used is described in more detail in [5].

The boundary conditions for the temperature at the boundaries of the cavity ensure the conservation of the internal energy of the liquid, i.e. average temperature in the cavity. Therefore, the maintenance of the average temperature was strictly controlled, i.e. implementation of relation (13) in the process of calculating was monitored.

\section{Numerical results}

The calculations were performed for the value of the Prandtl number $\operatorname{Pr}=7$. Based on the results of calculations, for each of the given values, the critical Grashof number was determined and the nonlinear supercritical regime was investigated in the region of up to several tens of supercriticalities. For the case of an infinitely elongated layer, i.e. for $L=\infty$, the quiescent state of the fluid (12) becomes unstable with respect to small normal disturbances when the Rayleigh number exceeds the critical value $R a_{c r}(\infty)=320$ defined in [1] and confirmed in [7,8]. Hence, it follows that the critical Grashof number is equal to $G r_{c r}(\infty)=45.7$ for an infinitely elongated cavity $L=\infty$ and the value of the Prandtl number considered in this paper.

Let us consider the effect of a smooth increase in the Grashof number on the stability of solution (12) corresponding to mechanical equilibrium. The finite horizontal size of the cavity, due to the presence of solid vertical walls that inhibit the development of disturbances, as a rule, leads to an increase in the critical numbers of the onset of convective instability. Therefore, the first series of calculations was carried out for the Grashof number $G r=70$ in the hope that it exceeds the as yet unknown critical number $G r_{c r}$ for $L=2$. Let us describe in more detail the methodology for this calculation. The following distributions were set as the initial state:

$$
\psi_{i, k}^{0}=0, \quad T_{i, k}^{0}=1-h \cdot k, \quad \varphi_{i, k}^{0}=\varphi^{c} .
$$

The initial value of the vorticity $\varphi^{c}$ at all grid nodes was assumed to be 10 . Calculations at lower absolute values $\varphi^{c}$, for example, at $\varphi^{c}=1$, led to the attenuation of the disturbance (14) and the establishment of an equilibrium state (12).

Fig. 1a shows the stationary solution obtained as a result of the numerical calculation of the evolution of the initial state (14) with $\varphi^{c}=10$. In subsequent calculations, to obtain a solution for other values of the Grashof number, the parameter continuation method was used, i.e. the stationary state obtained earlier for a certain Grashof number $G r_{1}$ was taken as the initial state, the Grashof number was changed per step $\Delta G r$, and by numerically solving the problem with this state taken as the initial one, a new stationary state for the Grashof number $G r_{2}=G r_{1}+\Delta G r$ was obtained. The step size for the Grashof number $\Delta G r$ varied from $\Delta G r=10$ to $\Delta G r=500$. Calculations have shown that such a step-by-step increase in the Grashof number from $G r=70$ to $G r=3000$ does not lead to a qualitative change in the structure of the supercritical flow, it remains single-vortex (see Fig. 1), although the intensity of movement increases many times. The temperature field changes so that a temperature gradient is formed along the horizontal boundaries, and the temperature gradient in the center of the cavity rotates clockwise by more than $90^{\circ}$. 


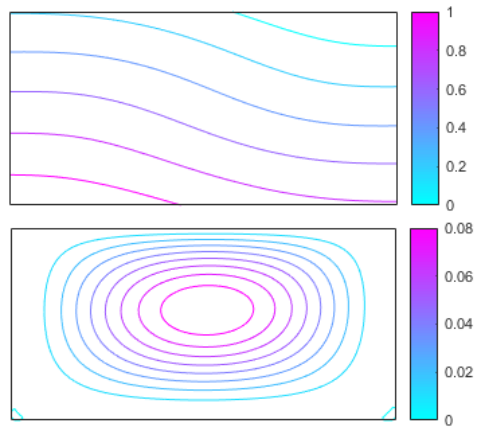

a)
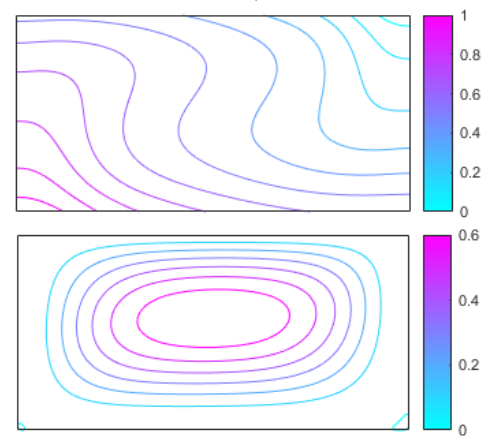

c)
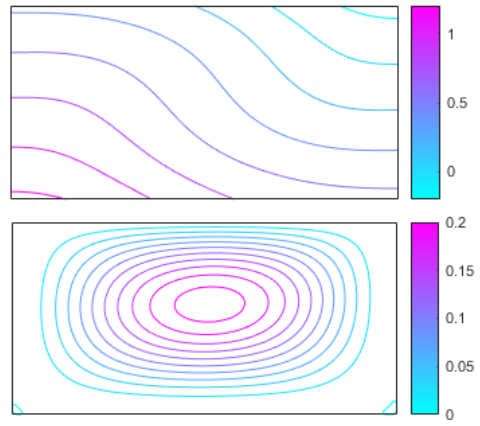

b)

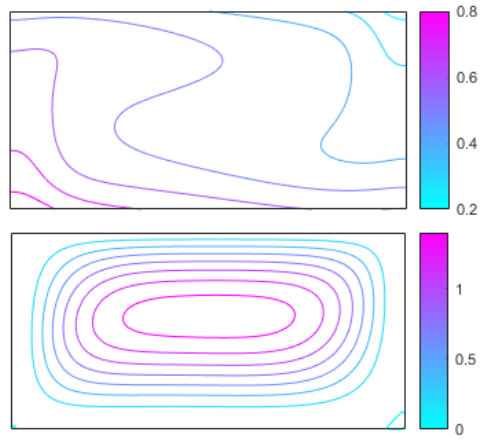

d)

Fig. 1. Isotherms and streamlines of the supercritical convective flow in the cavity $L=2$ for different values of the Grashof number: a) $-G r=70$, b) $-G r=100$, c) $-G r=500$, d) $-G r=3000$

To quantitatively characterize the change in the intensity of supercritical movements with an increase in the Grashof number, we will use the dependences of the average value of the stream function in the cavity $\psi_{s}(G r)$ and the kinetic energy of convective motion $E(G r)$ on $G r$ :

$$
\begin{gathered}
\psi_{s}(G r)=\frac{1}{L} \int_{0}^{1} \int_{0}^{L} \psi d z d x, \\
E(G r)=\frac{1}{2 L} \int_{0}^{1} \int_{0}^{L} v^{2} d z d x=\frac{1}{2 L} \int_{0}^{1} \int_{0}^{L} \psi \varphi d z d x .
\end{gathered}
$$

The dependences $\psi_{s}(G r)$ and $E(G r)$ obtained in the calculations and for the interval of Grashof numbers $0 \leqslant G r \leqslant 3000$ for the flow in the cavity $L=2$ are presented in Fig. 2 . In the region of Grashof numbers $70 \leqslant G r \leqslant 1000$, the kinetic energy of stationary solutions depends on the Grashof number linearly (the dependence is obtained by the least squares method):

$$
E=1.74 \cdot 10^{-3} \cdot(G r-64.5)
$$

Extrapolating linearly dependence (17) to zero $E=0$, we obtain the critical value of the Grashof number $G r_{c r}(2)=64.5$. In the area of Grashof numbers $70 \leqslant G r \leqslant 500$, the root law for the mean stream function is satisfied:

$$
\psi_{s}=1.17 \cdot 10^{-2} \cdot \sqrt{G r-64.5} \text {. }
$$


Dependencies (17) and (18) are shown by dashed lines in Fig.2.

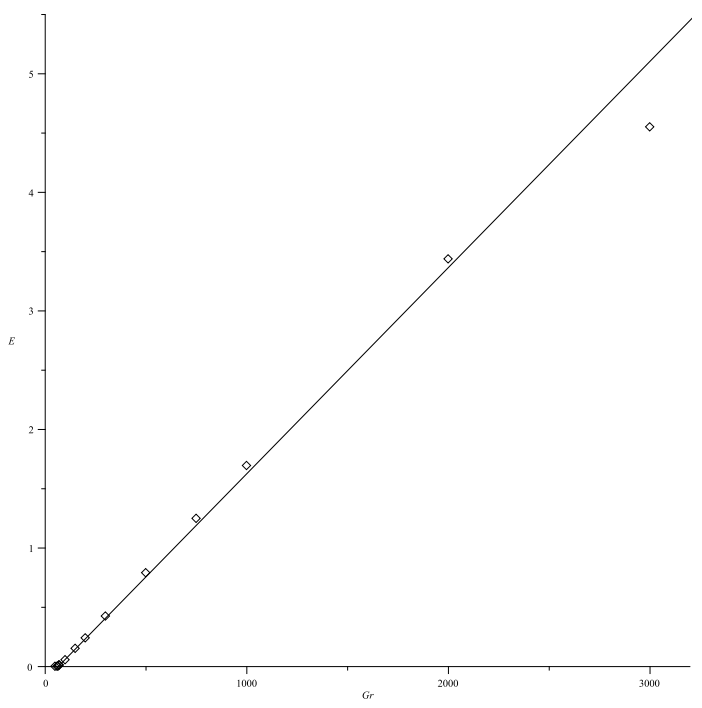

a)

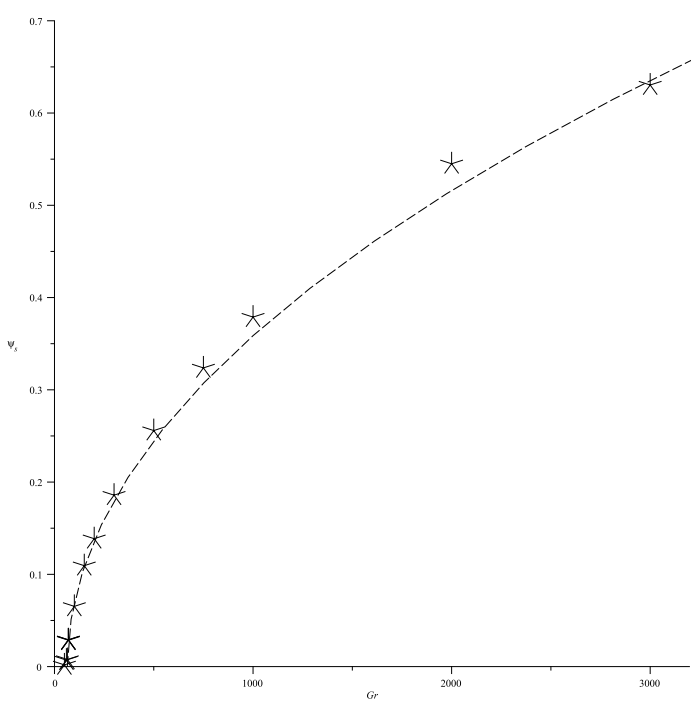

b)

Fig. 2. Dependences of kinetic energy $E(G r)$ (a) and average stream function $\psi_{s}(G r)$ (b) of stationary supercritical convective motion in the cavity $L=2$

Fig. 3a shows the stationary solution obtained as a result of the numerical calculation of the evolution of the initial state (14) with $\varphi_{c}=10$ for the case of a more elongated cavity $(L=3)$ than the one considered above. An increase in the elongation of the cavity on $50 \%$ does not lead to a qualitative change in the effect of an increase in the Grashof number on the structure of the supercritical flow (see Fig. 3).

The dependences $\psi_{s}(G r)$ and $E(G r)$ obtained in the calculations and for the interval of Grashof numbers $0 \leqslant G r \leqslant 5000$ for the motion shown in Fig. 3 are presented in Fig. 4 . In the region of Grashof numbers $70 \leqslant G r \leqslant 1000$, the kinetic energy of stationary motions depends on the Grashof number linearly:

$$
E=2.07 \cdot 10^{-3} \cdot(G r-61.1)
$$

Extrapolating this dependence linearly to zero, we obtain the critical value of the Grashof number $G r_{c r}=61.1$. In the area of Grashof numbers $70 \leqslant G r \leqslant 1000$, the root law for the stream function is satisfied:

$$
\psi_{s}=1.43 \cdot 10^{-2} \cdot \sqrt{G r-61.1}
$$

Dependencies (19) and (20) are shown by dashed lines in Fig. 4. The single-vortex flows obtained in the calculations are a manifestation of long-wave instability, since they can be interpreted as a result of the nonlinear development of the most horizontally extended perturbation. From the streamlines shown in Fig. 3d, it can be seen that for the case of a more elongated cavity, the development of this analogue of a two-wave disturbance leads to the formation of a plane-parallel advective flow in the central part of the cavity, on the upper and lower boundaries of which a constant horizontal temperature gradient is formed. 


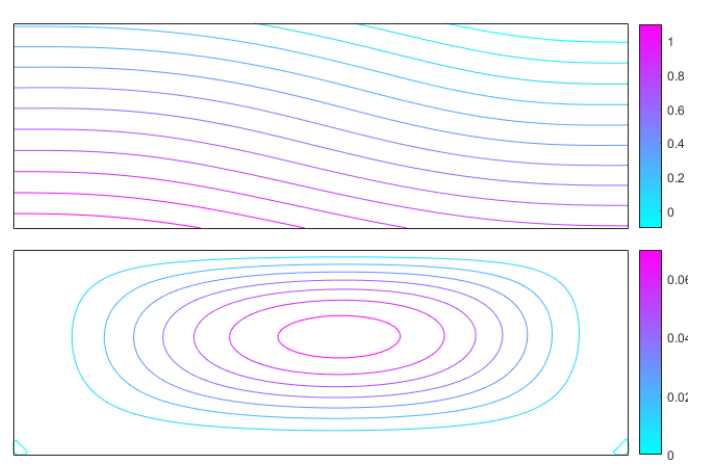

a)

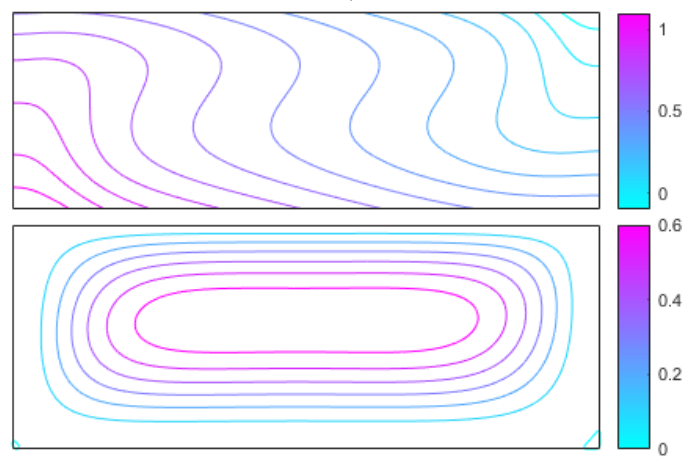

c)

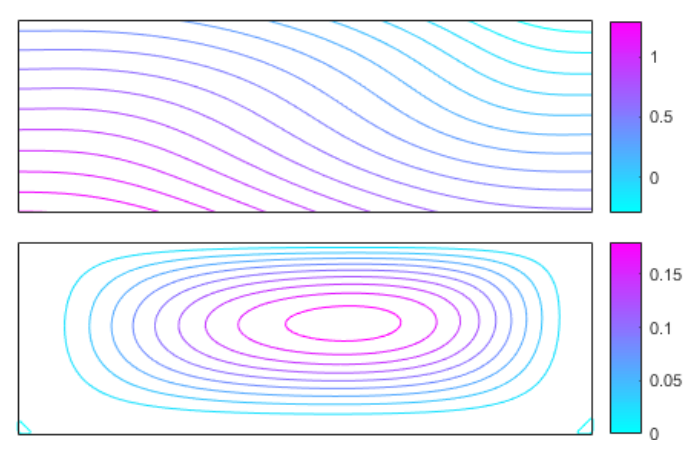

b)
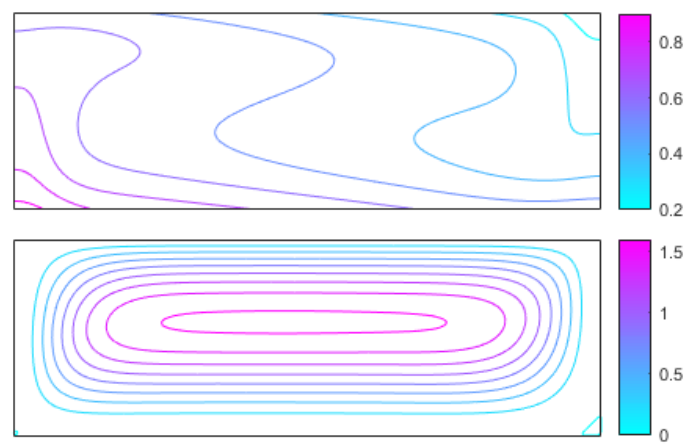

d)

Fig. 3. Isotherms and streamlines of the supercritical convective flow in the cavity $L=3$ for different values of the Grashof number: a) $-G r=70$, b) $-G r=100$, c) $-G r=500$, d) $-G r=3000$

\section{Conclusion}

Calculations have shown that even in moderately elongated cavities, the long-wave nature of convective instability manifests itself similarly to this in an infinite horizontal layer at a given vertical heat flux at the boundaries. A manifestation of the long-wave nature of the supercritical convection is that the supercritical convective flow in the entire considered interval of supercriticality has a large-scale one-vortex structure occupying the entire cavity. Note that with an increase in the intensity of heat transfer through the layer, the central part of the vortex is stretched, forming a plane-parallel flow. Such a plane-parallel flow in an infinite layer (i.e. at $L=\infty$ ), for the case of both rigid boundaries, can lose stability with respect to plane cellular disturbances $[9,10]$. Therefore, in future studies, the case when the horizontal size of the planeparallel flow will be sufficient for the appearance of cellular instability there will be calculated. It is also planned to take into account the thermocapillary effect at the free boundary, which can also lead to the appearance of cellular instability.

Calculations for $L=2$ were made within the framework of the state assignment. This study was funded by the Ministry of Science and High Education of Russian Federation (Grant No. FSNM-2020-0026). Calculations for $L=3$ were carried out with the support of the Russian Foundation for Basic Research (project 20-51-12010 NNIO_a) and NNIO (DFG: Projekt ZA $658 / 3-1)$. 


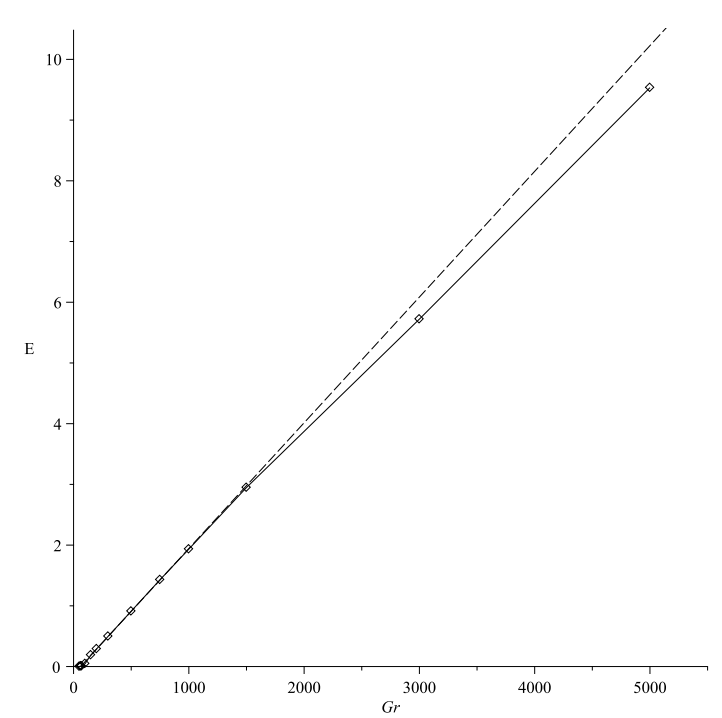

a)

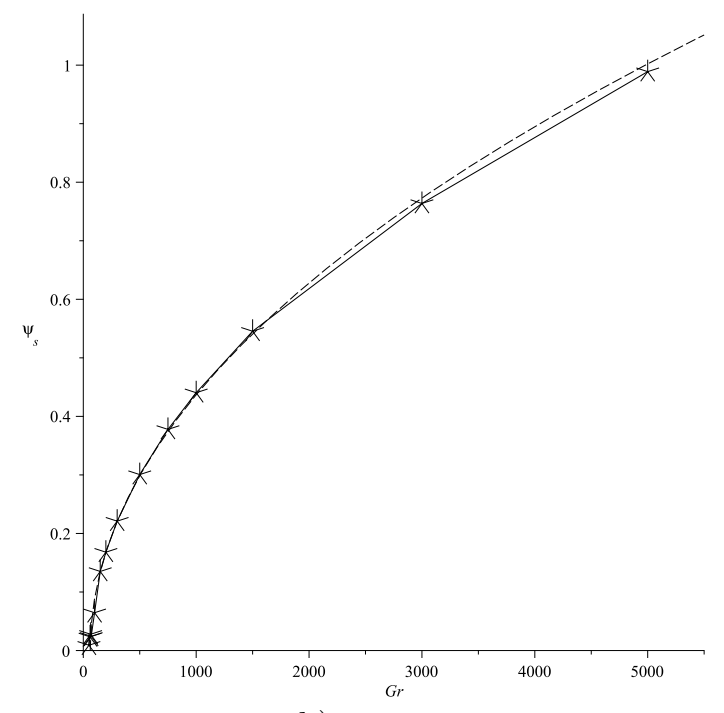

b)

Fig. 4. Dependences of the kinetic energy $E(G r)$ (a) and the average stream function $\psi_{s}(G r)$ (b) of the supercritical stationary convective flow in the cavity $L=3$

\section{References}

[1] E.M.Sparrow, R.J.Goldstein, V.K.Jonsson, Thermal instability in a horizontal fluid layer: effect of boundary conditions and non-linear temperature proille, Journal of Fluid Mechanics, 18(1964), no. 4. 513-528.

[2] L.Kh.Ingel, On the theory of slope flows, Journal of Engineering Physics and Thermophysics, 91(2018), no. 3, 641-647. DOI: 10.1007/s10891-018-1785-0

[3] V.B.Bekezhanova, Stability of the equilibrium state in a convection model with nonlinear temperature and pressure dependences of density, Journal of applied mechanics and technical physics, 48(2007), no. 2, 200-207

[4] T.P.Lyubimova, S.A.Prokopev, Nonlinear regimes of Soret-driven convection of ternary fluid with fixed vertical heat flux at the boundaries, The European Physical Journal E, 42(2019), no. 6, 76. DOI: 10.1140/epje/i2019-11837-4

[5] A.N.Sharifulin, Poludnitsin Anatoliy N. The borders of existence of anomalous convection flow in the inclined square cylinder: Numerical determination, St. Petersburg Polytechnical University Journal: Physics and Mathematics, 2(2016), no. 2, 150-156.

DOI: $10.1016 /$ j.spjpm.2016.05.013

[6] A.Thom, C.J.Apelt, Field computations in engineering and physics, 1961.

[7] D.V.Lyubimov, T.P.Lyubimova, V.A.Sharifulin, Onset of convection in a horizontal fluid layer in the presence of density inversion under given heat fluxes at its boundaries, Fluid Dynamics, 47(2012), no. 4, 448-453. DOI: 10.1134/S0015462812040035 
[8] V.A.Sharifulin, T.P.Lyubimova, Structure of critical perturbations in a horizontal layer of melted water with the prescribed heat flux at the boundaries, IOP Conf. Series: Materials Science and Engineering, Vol. 208, 2017, 012025.

[9] R.V.Sagitov, A.N.Sharifulin, Stability of advective flow in an inclined plane fluid layer bounded by solid planes with a longitudinal temperature gradient. 1. Unstable stratification, Journal of Applied Mechanics and Technical Physics, 58(2017), no. 2, 264-270.

DOI: $10.1134 /$ S0021894417020092

[10] R.V.Sagitov, A.N.Sharifulin, Stability of advective flow in an inclined plane fluid layer bounded by solid planes with a longitudinal temperature gradient 2. Stable stratification, Journal of Applied Mechanics and Technical Physics, 58(2017), no. 4, 687-692.

DOI: $10.1134 /$ S0021894417040137

\title{
Надкритическая конвекция воды в вытянутой полости при заданном вертикальном тепловом потоке
}

Вадим А. Шарифулин Пермский национальный исследовательский политехнический университет Пермь, Российская Федерация

Татьяна П. Любимова Институт механики сплошных сред УрО РАН

Пермь, Российская Федерация

\begin{abstract}
Аннотация. Исследуются надкритические режимы конвекции воды при комнатной температуре в вытянутых горизонтальных полостях, с отношением ширины к высоте $2: 1$ и $3: 1$. Число Прандтля полагается равным семи. На верхней свободной и нижней твердой границах задан постоянный тепловой поток, а боковые твердые границы полагаются теплоиз олированными. Расчеты, проведенные конечно-разностным методом для значений числа Релея, превышающих критическое до тридцати раз, показали, что в указанном интервале чисел Релея в обоих полостях в надкритической области реализуется одновихревое стационарное состояние.
\end{abstract}

Ключевые слова: тепловая конвекция, бифуркации, постоянный тепловой поток. 